University of New Hampshire

University of New Hampshire Scholars' Repository

\title{
$5-2012$
}

\section{The Impact of Sea State Condition on Airborne Lidar Bathymetry Measurements}

Torbjorn Karlsson

Lund Institute of Technology

Andreas Axelsson

Lund Institute of Technology

Shachak Pe'eri

University of New Hampshire, Durham, shachak.peeri@unh.edu

Follow this and additional works at: https://scholars.unh.edu/ccom

Part of the Oceanography and Atmospheric Sciences and Meteorology Commons

\section{Recommended Citation}

Torbjorn Karlsson ; Shachak Pe'eri and Andreas Axelsson, "The impact of sea state condition on airborne lidar bathymetry measurements", Proc. SPIE 8379, Laser Radar Technology and Applications XVII, 837913 (May 1, 2012); doi:10.1117/12.920608; http://dx.doi.org/10.1117/12.920608

This Conference Proceeding is brought to you for free and open access by the Center for Coastal and Ocean Mapping at University of New Hampshire Scholars' Repository. It has been accepted for inclusion in Center for Coastal and Ocean Mapping by an authorized administrator of University of New Hampshire Scholars' Repository. For more information, please contact Scholarly.Communication@unh.edu. 


\title{
The impact of sea state condition on Airborne Lidar Bathymetry measurements
}

\author{
Torbjorn Karlsson ${ }^{1,2}$, Shachak Pe'eri*3 ${ }^{3}$, Andreas Axelsson ${ }^{1}$ \\ ${ }^{1}$ Airborne Hydrography AB (AHAB), Jönköping, Sweden \\ ${ }^{2}$ Lund Institute of Technology, Lund University, Lund, Sweden \\ ${ }^{3}$ Center for Coastal and Ocean Mapping, University of New Hampshire, Durham, NH 03824
}

\begin{abstract}
Due to a large number of available Airborne Lidar Bathymetry (ALB) survey datasets and scheduled future surveys, there is a growing need from coastal mapping communities to estimate the accuracy of ALB as a function of the survey system and environmental conditions. Knowledge of ALB accuracy can also be used to evaluate the quality of products derived from ALB surveying. This paper presents theoretical and experimental results focused on the relationship between sea surface conditions and the accuracy of ALB measurements. The simulated environmental conditions were defined according to the typical conditions under which successful ALB surveys can be conducted. The theoretical part of the research included simulations, where the ray-path geometry of the laser beam was monitored below the water surface. Wave-tank experiments were conducted to support the simulations. A cross section of the laser beam was monitored underwater using a green laser with and without wind-driven waves. The results of the study show that capillary waves and small gravity waves distort the laser footprint. Because sea-state condition is related to wind at a first-order approximation, it is possible to suggest wind speed thresholds for different ALB survey projects that vary in accuracy requirements. If wind or wave information were collected during an ALB survey, then it is possible to evaluate the change in accuracy of ALB survey due to different sea surface conditions.
\end{abstract}

Key words: ALB, water surface, refraction, laser beam, ray tracing, wind speed, simulation

\section{INTRODUCTION}

In order to maintain safety of navigation in harbors, along channels and along the coastlines, mapping of the littoral zone should be conducted on a regular basis. The International Hydrographic Organization (IHO), an inter-governmental organization, has declared that safety of navigation is of great importance for commerce and for marine environmental protection [1]. The most common methods used today for shallow-water mapping are conventional acoustic technologies (single-beam, multibeam, and sweep systems) and airborne light detection and ranging (LIDAR) bathymetry (ALB). The choice between acoustic surveying or ALB depends on the survey. Mapping in waters deeper than $10 \mathrm{~m}$ is more suitable for acoustic systems. Shallow waters of depths less than $15 \mathrm{~m}$ that include areas dangerous for ship surveying are more suitable for aerial surveys. In addition, ALB can also survey over land, producing a seamless elevation map of the topography and bathymetry of the region.

ALB is a survey technology that is distinctly different from acoustic surveying. ALB is an active remote sensing technology that operates from a low altitude aircraft using a scanning pulsed laser beam [2]. The core of the ALB system is a Nd:YAG laser that transmits short green pulses at $532 \mathrm{~nm}$. The pulses are transmitted down into the water as laser beams using a scanner that controls the survey sweeping pattern. The peak returns from the interaction of the laser beams with the water surface and seafloor are collected back in the ALB system, where the time difference between the peaks is used to calculate the water depth. The location of each laser measurement is geo-referenced using the aircraft's (global positioning system) GPS and (inertial measurement unit) IMU systems [3]. The ALB's accuracy of measurement depends on the accuracy of the hardware components and environmental conditions at the time of survey. A clear understanding of both the hardware and the environment will help to assess the accuracy of the laser measurements, configure future ALB surveys, and improve the design of the ALB system for a better performance.

This paper presents a collaboration research between the Center for Coastal and Ocean Mapping (CCOM) at the University of New Hampshire (UNH), U.S.A, and Airborne Hydrography AB (AHAB), Sweden on the water surface as

Laser Radar Technology and Applications XVII, edited by Monte D. Turner, Gary W. Kamerman, Proc. of SPIE Vol. 8379, 837913 - @ 2012 SPIE · CCC code: 0277-786X/12/\$18 · doi: 10.1117/12.920608 
a source of error in the LIDAR measurements. Simulations and laboratory experiments have been conducted to monitor ray-path changes in the direction of the laser beam due to the ocean surface conditions. The study goals were to estimate the average direction and the variability of an ALB beam underwater and to estimate the beam pattern at a given water depth.

The parameters for the ALB beam were calculated based on the specifications of AHAB's HawkEye II system. Digital elevation maps (DEM) were developed to simulated ocean surfaces at different weather conditions. These DEMs were used to predict the laser beam interaction with a realistic ocean surfaces that are composed from a large number of facets with a broad spectrum of slopes and aspects. Ray-tracing simulations produced statistics on the ALB beam interaction with the water surface. In addition to the simulations, empirical measurement were conducted in the wave and tow tank facilities at UNH. These results were used to validate the simulation results.

\section{ACCURACY OF MEASUREMENT}

The ALB system calculates the water depth from the survey data using the laser waveforms. Short laser pulses (a few $\mathrm{nm}$ ) are transmitted from the system down towards the water. As the laser pulse interacts with air/water interface, the water surface, most of the energy is refracted down to the water column according to Snell's law of refraction. A small portion of the pulse energy is reflected back up and is collected by the ALB system.

As the laser beam passes through the water column, its cross-section expands with depth. The expansion is dependent on the beam divergence of the laser pulse, the cross-section area on the water surface and scattering in the water column. Depending on the water clarity, some amount of volume backscattering will occur. In shallow water depths, the laser pulse is able to reach the seafloor. The laser pulse energy interacts with the seafloor and is scattered back up through the water column. The scattering from the seafloor and the water column reflects some of the laser energy back up through the water column into the air and is collected by ALB system. A digitizer records the entire sequence of events from the interaction of the pulse with the water surface to the interaction with the seafloor as a waveform, where the received intensity (digital number) is logged as a function of time (nanoseconds). The distance of the laser pulse path is measured by determining the time difference between detection of the water surface and the water bottom. The water depth is calculated by correcting the distance of the laser-pulse path to its refracted angle on incidence underwater.

Although the operation concept of ALB is straight forward, the ALB design is complex. The main two challenges are: 1) recording the waveforms, and 2) synchronizing the waveforms to the position and attitude of the aircraft. The overall accuracy of the ALB measurement is compiled from all the accuracies of the different components in the system. In addition to the hardware, the environment also affects the accuracy. The main environmental factors include the water surface, the water column and the seafloor. Out of the three main environmental factors, very little has been discussed on the water surface. In ideal conditions, the water surface would be perfectly flat and only the off-nadir angle is needed to calculate the new direction of the refracted laser beam and amount of energy reflected back into the air.

However, the typical morphology of the sea surface is complex and highly variable in time and space. The water surface can be considered as a deformed lens that is refracting the light. As the water surface refracts the laser beam rays, it may focus or defocus them underwater. The uncertainty of the water surface will introduce errors in: 1) identifying the water surface in the waveform, 2) defining the location and the size of the illuminated area on the seafloor, and 3) knowing the refracted angle of the laser beam underwater to correct the distance of the laser pulse path for the water depth calculation.

\section{METHODOLOGY}

\subsection{ALB model}

The simulations and empirical measurements in the study were configured according to the hardware design and survey configuration of the AHAB HawkEye II system. The HawkEye system typically operates at altitudes of 300 to $400 \mathrm{~m}$ with a beam divergence of $14 \mathrm{mrad}$. The transmitted laser beam broadens proportional to its beam divergence, reaching a 
cross section area of 3 to $6 \mathrm{~m}$ in diameter on the water surface. The scanning pattern of Hawk Eye is controlled by a programmable scanner that permits a wide variety patterns, including a 20-degree off-nadir mode that is typical in bathymetric surveys. In this study, a survey pattern was assumed with an average footprint area of $4.5 \mathrm{~m}$ on the water surface. The laser beam was assumed to have a Gaussian cross section. It also assumed that apart from wind, the weather conditions over the study area were ideal for surveying and no scattering or absorption occurred between the ALB system (i.e., the airplane) and the sea surface.

\subsection{Water surface simulation}

Current models do not provide a complete description of the water surface for all wind conditions [4],[5]. The shape of the ocean changes constantly and the wavelength sizes of the wind-generated waves range from only a few millimeters up to several hundred meters or more [6]. Depending on the strength of the wind, different sizes of waves will be generated. Due to wave dispersion, waves at different lengths travel with different speeds. Another parameter affecting the speed of the waves, and also the shape of them, is the water depth [7]. Different groups of waves can interact with each other, giving rise to interference pattern that makes the model even more complicated. There is no single model that describes the water surface for all the environmental conditions. Instead, it is necessary to specify the environmental conditions in the study area. Those conditions should be as close as possible to the prevalent conditions when conducting ALB surveys.

The wavelength distribution of water-surface waves is controlled mainly by wind and water depth [4],[5]. The wind speed over the study area defines the wavelength distribution of the water surface waves. In shallow waters, the water depth modifies the wavelength distribution and changes the water surface pattern according to the bathymetry. In order to use "deep water" model, where the contribution from the bathymetry can be ignored, the shallowest water depth is assumed to be larger than half the wavelength of the largest wave generated by the wind [5]. Assuming that wind speed is lower than $5.4 \mathrm{~m} / \mathrm{s}$, this restriction on the bathymetry will exclude breaking waves that limit the ALB operation to map the seafloor. In addition, it is assumed that all waves are produced only by local wind and do not include waves that are produced outside the study area (e.g., swells). In this study, the Elfouhaily, Chapron, Katsaros, and Vandemark (ECKV) model [8] was used to generate the water surface. The general concept in water surface generation is applying a Fourier transform on the ECKV wave spectrum to convert the data from the frequency domain to a spatial domain and produce the water surface. Different wind conditions were used in the model, where a maximum wind speed of $5.4 \mathrm{~m} / \mathrm{s}(10.5 \mathrm{knt})$ was defined as the upper limit in the model. This value is based on the recommended ALB operational survey conditions [2]. The largest wave generated at these wind speeds is $40 \mathrm{~m}$ in amplitude, which restricts the survey area to water depth greater than $20 \mathrm{~m}$.

The theoretical simulations in the study are used to calculate the ray-path geometry of the refracted laser beam. It is not possible to simulate all the potential water surfaces that may occur during an ALB survey. Instead, the simulations can be used to develop a probability distribution function of the direction of the refracted rays that can describe the laserbeam rays after they interact with the water surface. The DEM of the water surface is a Triangulated Irregular Network (TIN) structure, consisting of millions of triangle facets. The water-surface models are then imported into the ray tracing program and used as the air/water interface to refract and reflect the laser beam. The number of rays used in the simulation is based on the ALB footprint area and the facet size. The wind speeds used in the ECKV model ranged from $3.0 \mathrm{~m} / \mathrm{s}$ to $5.0 \mathrm{~m} / \mathrm{s}$ and the fetch size ranged from $10 \mathrm{~km}$ to $10000 \mathrm{~km}$. The simulations included the extreme survey cases, where the azimuth of the laser beam and the wind direction are either aligned or perpendicular to each other. In addition, a second set of simulations was created in order to compare the empirical measurements to the theoretical model. The laser configuration and the surface wave parameters were all set according to laboratory experiments.

Based on a Gaussian cross section assumption, the laser rays that describe the ALB beam on the water surface are used as input in the simulation. Using Snell's law of refraction, the paths of the refracted rays were calculated. This simulation output (i.e., refracted rays) provides the average direction of the refracted beam and its expansion (scattering). A pattern of the beam can be produced at any depth by taking an underwater cross-section of the rays. The beam pattern from the simulation output can then be compared to empirical measurements. 
The empirical measurements were collected at the CCOM facilities, University of New Hampshire, Durham, NH in order to validate the simulation model in a well controlled environment. A continuous-wave $30 \mathrm{~mW}$ laser emitting at 532 $\mathrm{nm}$ was used to produce the laser beam into a $2.4 \mathrm{~m}$ deep wave and tow tank. Due to logistical constraints, the laser positioned at height of $4.14 \mathrm{~m}$ above the water surface and the beam divergence of the laser was expanded to $33 \mathrm{mrad}$ using external optics. Based on the HawkEye II system specifications, 20 degrees off-nadir angle was used in the experiments that produced a footprint of $0.14 \mathrm{~m}$ in diameter on the water surface. Surface waves were produced using a large fan that was placed just above the water surface. The fan provided a steady wind generating waves in the area of interest. The amplitudes of the surface waves were measured using a staff meter providing a voltage proportional to the wave height at a sampling rate of $200 \mathrm{~Hz}$. Instrumentation to measure the angular spreading of the waves was not available during the experiments. Instead, an angular spreading model was used for comparing the experiment results to the simulations.

A cross section of the laser beam was produced using a frosted acrylic sheet. The acrylic sheet was placed underwater below the laser beam at a tilted angle of 15 degrees. This position allowed starting the experiment with the refracted laser beam nadir to the sheet at flat water surface conditions. Still photos of the cross section were captured at $1 \mathrm{~Hz}$ using an underwater camera that was place behind the frosted sheet. The images were compared to cross-section images produced from the simulations.

\section{RESULTS}

In the simulations, the altitude of the ALB system was set to $300 \mathrm{~m}$ with a beam divergence of 14 mrad that generated a footprint with $2.25 \mathrm{~m}$ in radius. For computation efficiency purposes, a subset $8 \times 8 \mathrm{~m}^{2}$ grid with 5.4 million facets around the footprint area was used in the simulations from the water-surface grid generated from the ECKV spectrum model. A significant wave height was calculated from the generated surfaces and was compared to the wave height published in the literature under the same wind and fetch conditions. The results from the simulations showed that the slope distribution of the ocean surface has a significant impact on incident laser beam. The wind speeds in the ECKV spectrum models were limited up to $5.4 \mathrm{~m} / \mathrm{s}$ to prevent the sea from being fully developed with breaking waves. A right hand Cartesian coordinate system was used to describe the results, where the $\mathrm{x}$-axis is the wind direction, $\mathrm{z}$-axis is down and the y-axis is cross product. The distribution function of the facets composing the water surface showed similar characteristics for all wind and fetch conditions. The slope range of the facets in the $\mathrm{x}$-axis was always broader than the slope range in the y-axis.

The simulation results showed that wind speed and the footprint size were the main two parameters contributing to changes in the laser beam pattern underwater. Statistically, the mean direction of the laser beam over a large amount of realization ( $>1000$ realizations) does not deviate much from the refracted beam direction under a flat water surface. However, the standard deviation of the beam is defined based on the mean direction of all the refracted rays that compose the beam and are dependent on the water surface conditions. The standard deviation along the axis parallel to the wind direction, $\sigma_{\mathrm{x}}$, and along the axis perpendicular to the wind direction, $\sigma_{\mathrm{y}}$, correlates to the measurement uncertainty. As the standard deviation increases, it is harder to define the ray path geometry and pin point the location on the seafloor that is illuminated by the ALB. Also, more energy can scatter away from the collection field of view that reduces the signal to noise ratio.

The simulations showed that the standard deviation of the ALB beam increased as the wind speeds over the water surface increased. The standard deviation of the rays for a $4.5-\mathrm{m}$ in diameter beam increased from $\sigma_{\mathrm{x}}=0.30^{\circ}$ and $\sigma_{\mathrm{y}}=$ $0.18^{\circ}$ for a wind speed of $3 \mathrm{~m} / \mathrm{s}$ to $\sigma_{x}=0.84^{\circ}$ and $\sigma_{y}=0.49^{\circ}$ for a wind speed of $5.4 \mathrm{~m} / \mathrm{s}$, where the fetch length was maintained constant at $10,000 \mathrm{~km}$. The area of the ALB footprint over the water surface also affected the standard deviation. The standard deviation increased from $\sigma_{\mathrm{x}}=0.84^{\circ}$ and $\sigma_{\mathrm{y}}=0.49^{\circ}$ to $\sigma_{\mathrm{x}}=2.00^{\circ}$ and $\sigma_{\mathrm{y}}=1.63^{\circ}$ as the footprint size was reduced from $4.5-\mathrm{m}$ in diameter to an area of a single ray ( $>5 \mathrm{~mm}$ in diameter), respectively, while the wind speed $(5.4 \mathrm{~m} / \mathrm{s})$ and the fetch length $(10,000 \mathrm{~km})$ remained constant. According to the simulations, fetch lengths only partially modify the standard deviation. The spatial distribution of the surface facets broadens as the water surface starts to develop. The standard deviation of the rays for a $4.5-\mathrm{m}$ in diameter beam with a wind speed of $3.5 \mathrm{~m} / \mathrm{s}$ increases from 
$\sigma_{\mathrm{x}}=0.17^{\circ}$ and $\sigma_{\mathrm{y}}=0.10^{\circ}$ at a fetch length of $10 \mathrm{~km}$ to $\sigma_{\mathrm{x}}=0.39^{\circ}$ and $\sigma_{\mathrm{y}}=0.23^{\circ}$ at a fetch length of $100 \mathrm{~km}$. For larger fetch lengths, the standard deviation converges to a $\sigma_{\mathrm{x}}=0.44^{\circ}$ and $\sigma_{\mathrm{y}}=0.26^{\circ}$. The simulations also showed that the orientation of the laser beam with respect to the wind direction does not matter. The simulation results showed almost the same values for both extreme cases, where the laser beam is oriented perpendicular to the wind direction and parallel to the wind direction.

The results from empirical measurements supported the simulation results. Based on the wave heights logged by the staff meter, a wave spectrum of the water surface was calculated and was used to generate a water surface model. The results from the experiments and the simulations showed a good agreement in the ratio between the $\sigma_{\mathrm{x}}$ and $\sigma_{\mathrm{y}}$. This ratio agreement supported the choice of the angular spreading function to compare between the two datasets. The empirical measurements also showed that the orientation of the laser beam with respect to the wind direction does not matter. The only major difference in the results between the empirical measurements and the simulations is that the underwater imagery indicated that the mean direction of the laser beam was a deviating toward the fan (negative $\mathrm{x}$-direction). This might be due to the small diameter size of the footprint $(0.14 \mathrm{~m})$ or non-linear effects that have not been considered in the simulations.

\section{DISCUSSION}

This study has shown that there is a relationship of the laser beam pattern underwater to the water surface conditions. The water surface conditions are dependent on the wind speed, wind direction and fetch length. Although the spatial distribution of the facets on the water surface can change from one model to another, the parameters changing the underwater pattern of the ALB laser beam are the same. It is important to know the wind direction for estimating the standard deviations, but the orientation of the survey flight lines with respect to the wind does not affect the results. So in order to estimate the quality of a given (or planned) ALB survey, the operator will need to calculate the ALB footprint area on the water surface using the laser beam divergence and the survey flight altitude and estimate the water surface conditions from nowcasts (or forecasts) of the wind conditions. This quality estimation is only focused on one environmental factor. One should take into account also the water column conditions (water clarity and bubbles) and the seafloor (morphology and vegetation). The water column is an important factor because it can cause scattering and diffuse the refracted laser beam.

The simulation work presented here are preliminary results. More simulations using different water surface models and more empirical measurements need to be conducted for quantifying the reduction in accuracy of an ALB measurement based over a given water surface. The work should also include the interaction of the laser beam with the water column. With that said, the study results provide a useful tool for designing ALB systems and qualifying an ALB survey based on the system, survey configuration and weather conditions.

\section{CONCLUSIONS}

The accuracy of the ALB measurements and its bathymetric products depend on the hardware components and the environmental conditions at the time of survey. In this study, the impact of the sea surface condition on an ALB laser beam was evaluated using simulations and empirical measurements. The ALB hardware design used in the study was based on the AHAB's HawkEye II system and DEMs of ocean surfaces at different weather conditions were developed. Ray-tracing simulations were used to predict the laser beam interaction with ocean surfaces composed from a large number of facets with a broad spectrum of slopes and aspects. In addition to the simulations, empirical measurement were conducted in the wave and tow tank facilities at $\mathrm{UNH}$. These results were used to validate the simulation results.

In order to describe the water surface in a single model, a small subset of environmental conditions was used in the simulations. These conditions are defined to be as close as possible to the prevalent conditions when conducting ALB surveys. An ECKV model was used in the study to generate the wave spectrum for the water surface. The wind speeds used in the ECKV model to generate the wave spectrum ranged from $3.0 \mathrm{~m} / \mathrm{s}$ to $5.0 \mathrm{~m} / \mathrm{s}$ and the fetch size ranged from $10 \mathrm{~km}$ to $10000 \mathrm{~km}$ with no breaking waves. The largest wave generated in the model was $20 \mathrm{~m}$ in wavelength, where the shallowest water depth in the survey area was deeper than $40 \mathrm{~m}$. In addition, it is assumed that all waves are 
produced only by local wind and do not include waves that are produce outside the study area (e.g., swells). In addition to the simulation, empirical measurements were conducted in order to validate the simulation models in a well controlled environment. A second set of simulations was created using a wind speed (up to $4.5 \mathrm{~m} / \mathrm{s})$ and a laser footprint area $(0.14$ $\mathrm{m}$ in diameter) that were produced in the laboratory. Underwater cross-sections (beam patterns) were collected in the empirical measurements and compared to cross-section produced using the simulations.

The results from the simulations showed that the slope distribution of the ocean surface has a significant impact on incident laser beam. The simulation results showed that wind speed and the footprint size were the main two parameters contributing to changes in the laser beam pattern underwater. The slope distribution of the surface facets and the standard deviation distribution of the laser beam were broader along the axis parallel to the wind direction compared to the axis perpendicular to the wind in all the different wind speed and fetch cases. The beam divergence also contributed to the refraction. The simulations showed that the standard deviation of the ALB beam increased as the wind speeds over the water surface increased and/or the area of the ALB footprint over the water surface decreased. According to the simulations, fetch lengths only contribute in the early development of the sea surface. As the sea surface is in its more developed stages or fully developed, the changes in the standard deviation are small. The simulations also showed that the orientation of the laser beam with respect to the wind direction does not matter. However, the absolute direction of the wind is an important parameter in the generation process of water surface models. The results from empirical measurements supported the simulations results.

Although only preliminary results are available, this study can be viewed as a useful tool for designing ALB systems and qualifying an ALB survey based on the system, survey configuration and weather conditions. This study has shown that the two main parameters changing the underwater pattern of the ALB laser beam are water surface (wind speed and fetch) and the ALB footprint size that can be estimated before or after the survey.

\section{REFERENCES}

[1] International Hydrographic Office (IHO), "IHO Standards for Hydrographic Surveys", Special Pub. No. 44, February $5^{\text {th }}, 36$ pp. (2008).

[2] Maune, D. F., [The DEM Users Manual: Digital Elevation Model Technologies and Applications], ASPRS, Bethesda, MD, USA, 253-320 (2007).

[3] Baltsavias, E., "Airborne laser scanning: Basic relations and formulas," ISPRS J. Photogramm. Rem. Sens., 54 (3), 199-214 (1999).

[4] Komar, P. D., [Beach Processes and Sedimentation], Prentice Hall, 544 pp. (1998).

[5] Demirbilek, Z. and V. Linwood, [Coastal engineering manual], U.S. Army Corps of Engineers, USACE, 2002 engineer manual number: EM 1110-2-1100, Washington, DC (2002).

[6] Holthuijsen, L.H., [Waves in Oceanic and Coastal Waters], Cambridge University Press, 404 pp. (2007).

[7] Brown, E., A. Colling, D. Park D, J. Wright J., [Waves, Tides and Shallow-Water Processes], ButterworthHeinemann, Oxford, (2005).

[8] Elfouhaily, T., B. Chapron, K. Katsaros, D. Vandemark, "A unified directional spectrum for long and short winddriven waves”, Jour. Geophys. Res., 102 (C7), 15781-15796 (1997). 